\title{
Effective Prover for Minimal Inconsistency Logic
}

\author{
Adolfo Gustavo Serra Seca Neto and Marcelo Finger \\ Computer Science Department \\ Institute of Mathematics and Statistics \\ University of São Paulo \\ [adolfo,mfinger] @ime.usp.br
}

Summary. In this paper we present an effective prover for $\mathbf{m b C}$, a minimal inconsistency logic. The mbC logic is a paraconsistent logic of the family of logics of formal inconsistency. Paraconsistent logics have several philosophical motivations as well as many applications in Artificial Intelligence such as in belief revision, inconsistent knowledge reasoning, and logic programming. We have implemented the KEMS prover for $\mathbf{m b C}$, a theorem prover based on the $\mathbf{K E}$ tableau method for mbC. We show here that the proof system on which this prover is based is sound, complete and analytic. To evaluate the KEMS prover for $\mathbf{m b C}$, we devised four families of mbC-valid formulas and we present here the first benchmark results using these families.

\section{Introduction}

In this paper we present new theoretical and practical results concerning paraconsistent logics. On the theoretical side, we have devised a $\mathbf{K E}$ tableau method for $\mathbf{m b C}$, a minimal inconsistency logic, and proved that this proof system is correct, complete and analytic. And on the practical side, we have implemented a theorem prover based on the $\mathbf{m b C ~ K E}$ proof system and proposed a set of benchmarks for evaluating $\mathbf{m b C}$ provers.

Paraconsistent logics are tools for reasoning under conditions which do not presuppose consistency [3]. These logics have several philosophical motivations as well as many applications in Artificial Intelligence such as in belief revision [12], inconsistent knowledge reasoning [8], and logic programming [1].

The relevance of reasoning in the presence of inconsistent information can be seen in the following example ${ }^{l}$. Suppose we are working with classical logic and we have a theory (which is a set of formulas) $\Gamma$ such that $\Gamma \vdash A$ (i.e. from $\Gamma$ we can deduce $A$ ) and also $\Gamma \vdash \neg A$. That is, this theory allows us to reach two contradictory conclusions. Suppose also that $\Gamma \vdash B$. In classical logic, from $I \vdash A$ and $\Gamma \vdash \neg A$ we can derive $\Gamma \vdash C$ for any formula $C$. In particular, $\Gamma \vdash \neg B$.

\footnotetext{
${ }^{1}$ We assume familiarity with the syntax and semantics of propositional classical logic.
}

Please use the following format when citing this chapter:

Neto, A.G.S.S., Finger, M., 2006, in IFIP International Federation for Information Processing, Volume 217, Artificial Intelligence in Theory and Practice, ed. M. Bramer, (Boston: Springer), pp. 465-474. 
In classical logic, a contradictory theory is also trivial, therefore useless. Paraconsistent logics separate these concepts: a contradictory theory needs not to be trivial. Therefore, in a paraconsistent logic such as $\mathbf{m b C}$, one can have $\Gamma \vdash_{\mathbf{m b C}} A$, $\Gamma \vdash_{\mathbf{m b C}} \neg A$ and $\Gamma \vdash_{\mathbf{m b C}} B$ without necessarily having $\Gamma \vdash_{\mathbf{m b C}} \neg B$. Therefore, in paraconsistent logics one can have an inconsistent theory and still draw interesting conclusions from it.

There have been some implementations of paraconsistent formalisms $[1,4]$, but we do not know of any implementation of a special class of paraconsistency logics: logics of formal inconsistency (LFIs) [3]. This class internalizes the notions of consistency and inconsistency at the object-language level. We have extended the KEMS prover [11], originally developed for classical propositional logic, to deal with LFIs. The first version of this extension implements a tableau prover for $\mathbf{m b C}$, one of the simplest representatives of this class of logics. The KEMS prover for $\mathbf{m b C}$ is implemented in Java and AspectJ. Java is a well established object-oriented programming language and Aspect $J$ is the major representative of a new programming paradigm: aspect-oriented programming. Its source code available for download in $[10]$.

The KEMS prover is a KE-based Multi-Strategy theorem prover. The KE system, a tableau method developed by Marco Mondadori and Marcello D'Agostino [7], was presented as an improvement, in the computational efficiency sense, over the Analytic Tableau method [13]. A tableau system for $\mathbf{m b C}$ had already been presented in [3], but this system is more similar to analytic tableaux than to KE: it has five branching rules, which can lead to an inefficent implementation. And although this system is sound and complete it is not analytic. Therefore, to implement the KEMS prover for $\mathbf{m b C}$ we devised an $\mathbf{m b C ~ K E}$ system and obtained a sound, complete and analytic tableau proof system with only one branching rule.

To evaluate our prover correctness and performance, we needed some families of $\mathbf{~ m b C}$ problems. As we do not know any family of valid formulas elaborated specially for $\mathbf{m b C}$ or any paraconsistent logic, we devised four families of $\mathbf{m b C}$ valid problems for evaluating $\mathbf{m b C}$ provers. These families are not classically valid, since all of them use the non-classical consistency connective. With these families we obtained the first benchmark results for the KEMS mbC implementation.

\subsection{Outline}

In section 2 we present the $\mathbf{m b C}$ logic. The $\mathbf{m b C} \mathbf{K E}$ system is exhibited in section 3. There we also prove its analyticity, soundness and completeness. In section 4 we show the problem families we devised to evaluate $\mathbf{m b C}$ provers and in section 5 we present the results obtained with the KEMS prover for $\mathbf{m b C}$ using these families as benchmarks. Finally, in section 6 we draw some conclusions and point to future work.

\section{The mbC Logic}

The $\mathbf{m b C}$ logic is a member of the family of logics of formal inconsistency [3]. Logics of formal inconsistency are a class of paraconsistent logics that internalize the notions of consistency and inconsistency at the object-language level. Paraconsistent logics 
are tools for reasoning under conditions which do not presuppose consistency [3]. Formal characterizations of paraconsistent logics and logics of formal inconsistency can be found, respectively, in [9] and [3].

The logic $\mathbf{m b C}$ is the weakest ${ }^{2}$ LFI based on classical logic presented in [3]. It uses the same set of connectives as propositional classical logic (the binary connectives $\wedge, \vee, \rightarrow$, and the unary connective $\neg$ ), plus a new one: the unary consistency (o) connective. The intended reading of $\circ A$ is ' $A$ is consistent', that is, if $\circ A$ is true, $A$ and $\neg A$ are not both true. In $\mathbf{m b C}, \circ A$ is logically independent from $\neg(A \wedge \neg A)$, that is, $O$ is a primitive unary connective, not an abbreviation depending on conjunction and negation, as it happens in da Costa's $C_{n}$ hierarchy of paraconsistent logics [5]. Its axiomatization is shown below:

\section{Axiom schemas}

$$
\begin{aligned}
& A \rightarrow(B \rightarrow A) \\
& (A \rightarrow B) \rightarrow((A \rightarrow(B \rightarrow C)) \rightarrow(A \rightarrow C)) \\
& A \rightarrow(B \rightarrow(A \wedge B)) \\
& (A \wedge B) \rightarrow A \\
& (A \wedge B) \rightarrow B \\
& A \rightarrow(A \vee B) \\
& B \rightarrow(A \vee B) \\
& (A \rightarrow C) \rightarrow((B \rightarrow C) \rightarrow((A \vee B) \rightarrow C)) \\
& A \vee(A \rightarrow B) \\
& A \vee \neg A \\
& \circ A \rightarrow(A \rightarrow(\neg A \rightarrow B)) \\
& \quad \text { Inference rule }
\end{aligned}
$$

\section{Inference rule}

(Modus Ponens) $\frac{A, A \rightarrow B}{B}$

Now we present the formal definition of satisfiable and valid formulas in $\mathbf{m b C}$ [3]. Let $\mathbf{2} \stackrel{\text { def }}{=}\{0,1\}$ be the set of truth-values, where 1 denotes the 'true' value and 0 denotes the 'false' value. An mbC-valuation is any function $v:$ For $\longrightarrow \mathbf{2}$ subject to the following clauses:

$$
\begin{aligned}
& v(A \wedge B)=1 \text { iff } v(A)=1 \text { and } v(B)=1 \\
& v(A \vee B)=1 \text { iff } v(A)=1 \text { or } v(B)=1 ; \\
& v(A \rightarrow B)=1 \text { iff } v(A)=0 \text { or } v(B)=1 \\
& v(\neg A)=0 \text { implies } v(A)=1 \\
& v(\circ A)=1 \text { implies } v(A)=0 \text { or } v(\neg A)=0 .
\end{aligned}
$$

A formula $X$ is said to be satisfiable if truth-values can be assigned to its propositional variables in a way that makes the formula true, i.e. if there is at least one valuation such that $v(X)=1$. A formula is a valid if all possible valuations make the formula true. For instance, the formula $\neg(A \wedge \neg A \wedge \circ A)$ is a valid in mbC, while $\neg(A \wedge \neg A)$ is satisfiable.

\footnotetext{
${ }^{2}$ It is the weakest because all other LFIs presented in [3] prove more theorems.
} 


\section{A KE System for $\mathrm{mbC}$}

The Analytic Tableau method is probably the most studied tableau method. It was presented in [13] as "an extremely elegant and efficient proof procedure for propositional logic". The KE System, a tableau method developed by Marco Mondadori and Marcello D'Agostino [7], was presented as an improvement, in the computational efficiency sense, over the Analytic Tableau method. It is a refutation system that, though close to the Analytic Tableau method, is not affected by the anomalies of cut-free systems [6].

In [3], a sound and complete tableau proof system for $\mathbf{m b C}$ is presented. It was obtained by using a method introduced in [2]. This method is a generic method that automatically generates a set of tableau rules for certain logics. For mbC, the rules obtained for its binary connectives are the same as that from classical analytic tableaux. The system also has a branching rule (called $R_{b}$ ) similar to KE PB rule, as well as rules for negation $(\neg)$ and consistency $(0)$. In total, this tableau system has 5 branching rules.

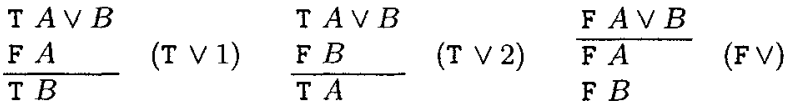

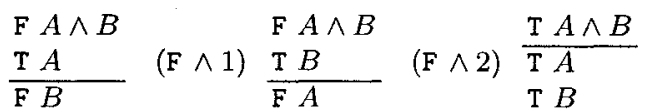

$$
\begin{aligned}
& \begin{array}{l}
\mathrm{T} A \rightarrow B \\
\frac{\mathrm{T} A}{\mathrm{~T} B} \quad(\mathrm{~T} \rightarrow 1) \frac{\mathrm{T} A \rightarrow B}{\frac{\mathrm{F} B}{\mathrm{~F} A}} \quad(\mathrm{~T} \rightarrow 2) \frac{\mathrm{F} A \rightarrow B}{\mathrm{~T} A} \\
\mathrm{~F} B
\end{array} \quad(\mathrm{~F} \rightarrow) \\
& \begin{array}{l}
\mathrm{T} \neg A \\
\frac{\mathrm{T} \circ A}{\mathrm{~F} A} \quad(\mathrm{~T} \neg)
\end{array} \quad \frac{\mathrm{F} \neg A}{\mathrm{~T} A} \quad(\mathrm{~F} \neg) \\
& \overbrace{\mathrm{T} A}
\end{aligned}
$$

Fig. 1. mbC KE tableau expansion rules

As explained in [6], branching rules lead to inefficiency. To obtain a more efficient proof system, we devised an original $\mathbf{~ m b C ~ K E ~ s y s t e m ~ u s i n g ~ s i g n e d ~ f o r m u l a s ~ ( s e e ~}$ Figure 1). A signed formula is an expression $S X$ where $S \in\{\mathrm{T}, \mathrm{F}\}$ is called the sign and $X$ is a propositional formula. The symbols $\mathrm{T}$ and $\mathrm{F}$, respectively representing 
the truth-values true and false, can be used as signs. The conjugate of a signed formula $\mathrm{T} A$ (or $\mathrm{F} A$ ) is $\mathrm{F} A$ (or $\mathrm{T} A$ ). The $\mathrm{mbC}(\mathrm{T} \neg$ ) rule is a LFI version of classical propositional logic $(\mathrm{T} \neg)[6]$. It states clearly that in $\mathrm{mbC}$ we need $\mathrm{T} \neg A$ and $\mathrm{T} \circ A$ to obtain $\mathrm{F} A$. In classical logic, we can obtain $\mathrm{F} A$ directly from $\mathrm{T} \neg A$.

\subsection{Analyticity, Correctness and Completeness Proof for the mbC KE system}

An mbC KE proof enjoys the subformula property if every signed formula in the proof tree is a subformula of some formula in the list of signed formulas to be proved. Let us call analytic the applications of PB which preserve the subformula property, and the analytic restriction of $\mathbf{m b C ~} \mathbf{K E}$ the system obtained by restricting $\mathrm{PB}$ to analytic applications. Given a rule $R$ of an expansion system $\mathrm{S}$, we say that an application of $R$ to a branch $\theta$ is analytic when it has the subformula property, i.e. if all the new signed formulas appended to the end of $\theta$ are subformulas of signed formulas occurring in $\theta$. According to [6], a rule $R$ is analytic if every application of it is analytic. It is easy to notice that all $\mathbf{m b C} \mathbf{K E}$ rules except (PB) are analytic.

We prove here that the $\mathbf{m b C} \mathbf{K E}$ system is analytic, sound and complete (some proofs were omitted due to lack of space). It is easy to show a procedure that transforms any proof in the original tableau system for $\mathbf{m b C}([3])$ in an $\mathbf{~ m b C ~ K E}$ proof, thus proving that $\mathbf{~ m b C ~ K E ~ s y s t e m ~ i s ~ a l s o ~ s o u n d ~ a n d ~ c o m p l e t e . ~ W e ~ w i l l ~ n o t ~ d o ~}$ this here. Instead, we will demonstrate that even the analytic restriction of $\mathbf{m b C ~ K E}$ is sound and complete. That is, when performing a proof we can restrict ourselves to analytic applications of $\mathrm{PB}$, applications which do not violate the subformula property, without affecting completeness.

The proof will be as follows. First we will redefine the notion of downward saturatedness for $\mathbf{m b C}$. Then we will prove that every downward saturated set is satisfiable. The mbC KE proof search procedure for a set of signed formulas $S$ either provides one or more downward saturated sets that give a valuation satisfying $S$ or finishes with no downward saturated set. Therefore, if an mbC KE tableau for a set of formulas $S$ closes, then there is no downward saturated set that includes it, so $S$ is unsatisfiable. However, if the tableau is open and completed, then any of its open branches can be represented as a downward saturated set and be used to provide a valuation that satisfies $S$. By construction, downward saturated sets for open branches are analytic, i.e. include only subformulas of $S$. Therefore, the mbC KE system is analytic. As a corollary, it is also sound and complete.

Definition 1. A set of signed formulas DS is downward saturated if

1. whenever a signed formula is in $D S$, its conjugate is not in $D S$;

2. when all premises of any $\mathbf{m b C} \mathbf{K E}$ rule (except $\mathrm{PB}$ ) are in $D S$, its conclusions are also in $D S$;

3. when the major premise of a mbC KE rule is in $D S$, either its auxiliary premise or its conjugate is in $D S$.

For $\mathbf{m b C ~ K E}$, item (3) above is valid for every rule except $(T \neg)$. In this case, if $\mathrm{T} \neg X$ is in $D S$, either $\mathrm{T} \circ X$ or $\mathrm{F} \circ X$ is in $D S$ only if $\circ X$ is a subformula of some other formula in $D S$. 
We extend valuations to signed formulas in an obvious way: $v(\mathrm{~T} A)=v(A)$ and $v(\mathrm{~F} A)=1-v(A)$. A set of signed formulas $L$ is satisfiable if it is not empty and there is a valuation such that for every formula $\mathcal{S} X \in L, v(\mathcal{S} X)=1$. Otherwise, it is unsatisfiable.

Lemma 1. (Hintikka's Lemma) Every downward saturated set is satisfiable.

Proof. For any downward saturated set $D S$, we can easily construct a valuation $v$ such that for every signed formula $\mathcal{S} X$ in the set, $v(\mathcal{S} X)=1$. How can we guarantee this is in fact a valuation? First, we know that there is no pair T $X$ and $F X$ in $D S$. Second, mbC KE rules preserve valuations. That is, if $v\left(\mathcal{S} X_{i}\right)=1$ for every premise $\mathcal{S} X_{i}$, then $v\left(\mathcal{S} C_{j}\right)=1$ for all conclusions $C_{j}$. And if $v\left(\mathcal{S} X_{1}\right)=1$ and $v\left(\mathcal{S} X_{2}\right)=0$, where $X_{1}$ and $X_{2}$ are, respectively, major and minor premises of an $\mathbf{m b C ~ K E}$ rule, then $v\left(\mathcal{S}^{\prime} X_{2}\right)=1$, where $\mathcal{S}^{\prime} X_{2}$ is the conjugate of $\mathcal{S} X_{2}$. For instance, suppose T $A \wedge B \in D S$, then $v(\mathrm{~T} A \wedge B)=1$. In accord with the definition of downward saturated sets, $\{\mathrm{T} A, \mathrm{~T} B\} \subseteq D S$. And by the definition of valuation, $v(\mathrm{~T} A \wedge B)=1$ implies $v(\mathrm{~T} A)=v(\mathrm{~T} B)=1$.

Theorem 1. $D S^{\prime}$ is a set of signed formulas. $D S^{\prime}$ is satisfiable if and only if there exists a downward saturated set $D S^{\prime \prime}$ such that $D S^{\prime} \subseteq D S^{\prime \prime}$.

Corollary 1. $D S^{\prime}$ is a unsatisfiable set of formulas if and only if there is no downward saturated set $D S^{\prime \prime}$ such that $D S^{\prime \prime} \subseteq D S^{\prime}$.

Theorem 2. The mbC KE system is analytic.

Proof. The mbC KE proof search procedure for a set of signed formulas $S$ either provides one or more downward saturated sets that give a valuation satisfying $S$ or finishes with no downward saturated set. If an $\mathbf{~ m b C ~ K E ~ t a b l e a u ~ f o r ~ a ~ s e t ~ o f ~}$ formulas $S$ closes, then there is no downward saturated set that includes it, so $S$ is unsatisfiable. If the tableau is open and completed, then any of its open branches can be represented as a downward saturated set and be used to provide a valuation that satisfies $S$. By construction, downward saturated sets for open branches are analytic, i.e. include only subformulas of $S$. Therefore, the $\mathbf{m b C}$ KE system is analytic.

Corollary 2. The mbC KE system is sound and complete.

\section{Problem Families}

We present below the problem families we devised to evaluate $\mathbf{m b C}$ theorem provers. We had two objectives in mind. First, to obtain families of $\mathbf{m b C}$-valid problems whose $\mathbf{m b C}$ KE proofs were as complex as possible. And second, to devise problems which required the use of many, if not all, mbC KE rules. These families are not classically valid, since all of them have formulas with the non-classical consistency connective. 


\subsection{First family}

Here we present the first family $\left(\Phi^{1}\right)$ of valid sequents for mbC. In this family all $\mathbf{m b C}$ connectives are used. It is easy to obtain polynomial $\mathbf{m b C}$ KE proofs for this family of problems. The sequent to be proved for the $n$-th instance of this family $\left(\Phi_{n}^{1}\right)$ is:

$$
\bigwedge_{i=1}^{n}\left(\neg A_{i}\right), \bigwedge_{i=1}^{n}\left(\left(\circ A_{i}\right) \rightarrow A_{i}\right),\left[\bigvee_{i=1}^{n}\left(\circ A_{i}\right)\right] \vee\left(\neg A_{n} \rightarrow C\right) \vdash C
$$

The explanation for this family is as follows. Suppose we are working with a database that allows inconsistent information representation. $A_{i}$ means that someone expressed an opinion $A$ about an individual $i$ and $\neg A_{i}$ means that someone expressed an opinion $\neg A$ about this same individual. For instance, if $A$ means that a person is nice, $\neg A_{3}$ means that at least one person finds 3 is not nice, and $A_{4}$ means that at least one person finds 4 nice. Then $\circ A_{i}$ means that either all people think $i$ is nice, or all people think $i$ is not nice, or there is no opinion $A$ recorded about $i . \circ A_{i} \rightarrow A_{i}$ means that if all opinions about a person are the same, then that opinion is $A$.

For a subset of individuals numbered from 1 to $n$, we have $\neg A_{i}$ and $\circ A_{i} \rightarrow A_{i}$ for all of them. From the fact that either $\neg A_{n} \rightarrow C$ or for one of them we have $\circ A_{i}$, we can conclude $C$.

\subsection{Second Family}

The second family of problems for $\mathbf{m b C}\left(\Phi^{2}\right)$ is a variation over the first family whose proofs are exponential in size. The sequent to be proved for the $n$-th instance of this family $\left(\Phi_{n}^{2}\right)$ is:

$$
\begin{aligned}
& \bigwedge_{i=1}^{n}\left(\neg A_{i}\right),\left[\bigwedge_{i=1}^{n}\left[\left(\circ A_{i}\right) \rightarrow\left(\left[\bigvee_{j=i+1}^{n} \circ A_{j}\right] \vee\left(\left(\neg A_{n}\right) \rightarrow C\right)\right)\right]\right] \\
& {\left[\bigvee_{i=1}^{m}\left(\circ A_{i}\right)\right] \vee\left(\neg A_{n} \rightarrow C\right) \vdash C}
\end{aligned}
$$

This family is a modification of the first family where instead of a conjunction of $\circ A_{i} \rightarrow A_{i}$, we have a conjunction of $\circ A_{i} \rightarrow\left(\left[\bigvee_{j=i+1}^{n} \circ A_{j}\right] \vee\left(\left(-A_{n}\right) \rightarrow C\right)\right)$ meaning that for every person numbered 1 to $n$, if all opinions about a person are the same, then either all opinions about some other person with a higher index are the same or $\left(\neg A_{n}\right) \rightarrow C$ is true.

\subsection{Third Family}

With the third family of problems we intended to develop a family whose instances required the application of all $\mathbf{m b C} \mathbf{K E}$ rules. To devise the third family $\left(\Phi^{3}\right)$, we have made some changes to the second family trying to make it more difficult to prove. The $n$-th instance of this family $\left(\Phi_{n}^{3}\right)$ is the following sequent: 


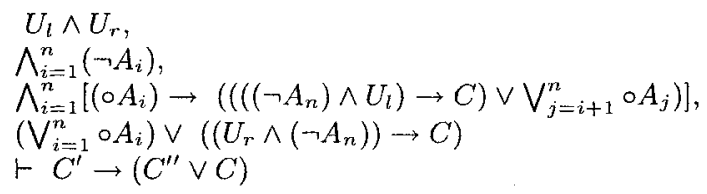

\subsection{Fourth Family}

This is the only of these families where negation appears only in the conclusion. The $n$-th instance of this family $\left(\Phi_{n}^{4}\right)$ is the following sequent:

$$
\bigwedge_{i=1}^{n}\left(A_{i}\right), \bigwedge_{j=1}^{n}\left(\left(A_{j} \vee B_{j}\right) \rightarrow\left(\circ A_{j+1}\right)\right),\left[\bigwedge_{k=2}^{n}\left(\circ A_{k}\right)\right] \rightarrow A_{n+1} \vdash \neg \neg A_{n+1}
$$

Note: if $n \leq 2,\left[\bigwedge_{i=2}^{n}\left(\circ A_{i}\right)\right]$ in $\left[\bigwedge_{i=2}^{n}\left(\circ A_{i}\right)\right] \rightarrow A_{n+1}$ is replaced by the $T$ formula.

This family formulas can be explained as follows. We have two formulas to represent two types of opinion: $A$ and $B$. First we assume $A_{i}$ for every $i$ from 1 to $n$. Then we suppose for all $j$ from 1 to $n$ that $\left(A_{j} \vee B_{j}\right)$ implies $\circ A_{j+1}$. And finally we assume that for every $k$ from 2 to $n$ the conjunction of $A_{k}$ 's implies $A_{n+1}$. It is easy to see that from these assumptions we can deduce $A_{n+1}$. So we can also deduce its double negation: $\neg \neg A_{n+1}$.

\section{Evaluation}

Theorem provers are usually compared by using benchmarks. We have extended KEMS prover [11] to prove mbC theorems and evaluated it using as benchmarks the problem families presented in section 4 . In Table 1 we show some of the results obtained. The tests were run on a personal computer with an Athlon 1100Mhz processor, $384 \mathrm{Mb}$ of memory, running a Linux operating system with a 2.26 kernel.

\begin{tabular}{|c|c|c|c|c|}
\hline Problem & Time spent $(\mathrm{s})$ & Problem size & Proof size & e Tree height \\
\hline$\Phi_{4}^{1}$ & 0.06 & 47 & 197 & 4 \\
\hline$\Phi_{7}^{\hat{1}}$ & 0.046 & 80 & 491 & 7 \\
\hline$\Phi_{10}^{1}$ & 0.08 & 113 & 911 & 10 \\
\hline$\Phi_{4}^{2}$ & 0.071 & 77 & 570 & 7 \\
\hline$\Phi_{7}^{2}$ & 1.54 & 164 & 7350 & 13 \\
\hline$\Phi_{10}^{2}$ & 21.964 & 278 & 116037 & 19 \\
\hline$\Phi_{4}^{3}$ & 0.058 & 94 & 706 & $\overline{6}$ \\
\hline$\Phi_{7}^{3}$ & 1.097 & 187 & 5432 & 9 \\
\hline$\Phi_{10}^{3}$ & 17.595 & 307 & 52540 & 12 \\
\hline$\overline{\Phi_{4}^{4}}$ & 0.007 & 47 & 181 & 3 \\
\hline$\Phi_{7}^{4}$ & 0.013 & 83 & 433 & 3 \\
\hline$\Phi_{10}^{4}$ & 0.023 & 119 & 793 & 3 \\
\hline
\end{tabular}

Table 1. KEMS results for $\mathbf{m b C}$ 
From these results it is clear that the second and third families are much more difficult to prove than the other two. And interestingly enough it was easier to prove the third than the second family.

\section{Conclusion}

We have presented an effective prover for mbC: a minimal inconsistency logic. The mbC KE system it implements was proven to be sound, complete and analytic. Besides that, it has only one branching rule. We devised some families of valid problems to evaluate our prover correctness and performance. These families can be used to evaluate any $\mathbf{m b C}$ theorem prover. The KEMS prover for $\mathbf{m b C}$ obtained the first benchmark results for these problem families.

In the future we intend to design different KEMS strategies for mbC. For instance, we want to implement a strategy that uses some derived rules not presented here. After that, we want to extend the KEMS prover to deal with $C_{1}$, the first logic in da Costa's $C_{n}$ hierarchy of paraconsistent logics [5].

This paper has been partially sponsored by FAPESP Thematic Project Grant ConsRel 2004/14107-2.

\section{References}

1. H. A. Blair and V. S. Subrahmanian. Paraconsistent logic programming. Theor. Comput. Sci., 68(2):135-154, 1989.

2. Carlos Caleiro, Walter Carnielli, Marcelo E. Coniglio, and Joao Marcos. Two's company: "The humbug of many logical values". In Logica Universalis, pages 169-189. Birkhäuser Verlag, Basel, Switzerland, 2005.

3. Walter Carnielli, Marcelo E. Coniglio, and Joao Marcos. Logics of Formal Inconsistency. In Handbook of Philosophical Logic, volume 12. Kluwer Academic Publishers, 2005.

4. Newton C. A. da Costa, Lawrence J. Henschen, James J. Lu, and V. S. Subrahmanian. Automatic theorem proving in paraconsistent logics: theory and implementation. In CADE-10: Proceedings of the tenth international conference on Automated deduction, pages 72-86, New York, NY, USA, 1990. SpringerVerlag New York, Inc.

5. Newton C. A. da Costa, Décio Krause, and Otávio Bueno. Paraconsistent logics and paraconsistency: Technical and philosophical developments. CLE e-prints (Section Logic), 4(3), 2004.

6. Marcello D'Agostino. Tableau methods for classical propositional logic. In Marcello D'Agostino et al., editor, Handbook of Tableau Methods, chapter 1, pages 45-123. Kluwer Academic Press, 1999.

7. Marcello D'Agostino and Marco Mondadori. The taming of the cut: Classical refutations with analytic cut. Journal of Logic and Computation, pages 285-319, 1994.

8. Sandra de Amo, Walter Alexandre Carnielli, and Joao Marcos. A logical framework for integrating inconsistent information in multiple databases. In FoIKS 
'02: Proceedings of the Second International Symposium on Foundations of Information and Knowledge Systems, pages 67-84, London, UK, 2002. SpringerVerlag.

9. Itala M. Loffredo D'Ottaviano and Milton Augustinis de Castro. Analytical Tableaux for da Costa's Hierarchy of Paraconsistent Logics $C_{n}, 1 \leq n \leq \omega$. Journal of Applied Non-Classical Logics, 15(1):69-103, 2005.

10. Adolfo Gustavo Serra Seca Neto. KEMS - A KE-based MultiStrategy Theorem Prover, 2006. Retrieved February 01, 2006, from http://gsd.ime.usp.br/ adolfo/projetos/KEMS.zip.

11. Adolfo Gustavo Serra Seca Neto and Marcelo Finger. Implementing a multistrategy theorem prover. In Ana Cristina Bicharra Garcia and Fernando Santos Osório, editors, Proceedings of the V ENIA, São Leopoldo-RS, Brazil, July 22-29 2005, 2005.

12. G. Priest. Paraconsistent Belief Revision. Theoria, 67:214-228, 2001.

13. Raymond M. Smullyan. First-Order Logic. Springer-Verlag, 1968. 